\title{
Combined medical and surgical treatment after acute spinal cord injury: results of a prospective pilot study to assess the merits of aggressive medical resuscitation and blood pressure management
}

\author{
Fernando L. Vale, M.D., Jennifer Burns, M.D., Amie B. Jackson, M.D., and Mark N. Hadley, M.D. \\ Division of Neurosurgery and Department of Rehabilitative Medicine, University of Alabama at \\ Birmingham, Birmingham, Alabama
}

The optimal management of acute spinal cord injuries remains to be defined. The authors prospectively applied resuscitation principles of volume expansion and blood pressure maintenance to 77 patients who presented with acute neurological deficits as a result of spinal cord injuries occurring from C-1 through T-12 in an effort to maintain spinal cord blood flow and prevent secondary injury. According to the Intensive Care Unit protocol, all patients were managed by Swan-Ganz and arterial blood pressure catheters and were treated with immobilization and fracture reduction as indicated. Intravenous fluids, colloid, and vasopressors were administered as necessary to maintain mean arterial blood pressure above $85 \mathrm{~mm} \mathrm{Hg}$. Surgery was performed for decompression and stabilization, and fusion in selected cases. Sixty-four patients have been followed at least 12 months postinjury by means of detailed neurological assessments and functional ability evaluations.

Sixty percent of patients with complete cervical spinal cord injuries improved at least one Frankel or American Spinal Injury Association (ASIA) grade at the last follow-up review. Thirty percent regained the ability to walk and $20 \%$ had return of bladder function 1 year postinjury.

Thirty-three percent of the patients with complete thoracic spinal cord injuries improved at least one Frankel or ASIA grade. Approximately $10 \%$ of the patients regained the ability to walk and had return of bladder function.

As of the 12-month follow-up review, $92 \%$ of patients demonstrated clinical improvement after sustaining incomplete cervical spinal cord injuries compared to their initial neurological status. Ninety-two percent regained the ability to walk and $88 \%$ regained bladder function.

Eighty-eight percent of patients with incomplete thoracic spinal cord injuries demonstrated significant improvements in neurological function 1 year postinjury. Eighty-eight percent were able to walk and $63 \%$ had return of bladder function.

The authors conclude that the enhanced neurological outcome that was observed in patients after spinal cord injury in this study was in addition to, and/or distinct from, any potential benefit provided by 
surgery. Early and aggressive medical management (volume resuscitation and blood pressure augmentation) of patients with acute spinal cord injuries optimizes the potential for neurological recovery after sustaining trauma.

\section{Key Words * spinal cord injury * trauma * quadriplegia * paraplegia * neurological recovery * ambulation}

The optimal treatment of acute spinal cord injuries remains to be elucidated. Advancements in the care of patients with acute spinal column injuries who have sustained neurological deficits include early immobilization to prevent further motion injury, rapid triage and transport from the geographic location at which the injury occurred to a definitive care facility, and administration of pharmacological agents to prevent secondary injury to remaining viable spinal cord tissue.[2,3,12] Early realignment of spinal fracture-dislocation injuries and early operative decompression performed in patients with spinal cord compression due to displaced bone, disc material, or a hematoma appear to have merit.[1,13,15,32,40] Despite clinical and experimental data indicating that early decompression improves outcome, $[6,14,26]$ early surgical intervention has not been conclusively demonstrated to be of significant clinical benefit. $[9,18]$

Early aggressive volume resuscitation and the elevation of a patient's mean arterial blood pressure (MABP) to supranormal levels have proved beneficial in patients suffering from cerebral ischemia due to subarachnoid hemorrhage, stroke, and closed head injury. Patients managed with volume expansion and hypertensive therapy have improved neurological outcomes compared with similar patients treated without aggressive medical resuscitation.[24,25,27-29] Although this form of therapy improves cerebral perfusion and cerebral blood flow after head injury, acute, aggressive medical resuscitation with elevation of the MABP is not a recognized or routinely recommended form of treatment in the patient with a spinal cord injury following acute trauma. However, as the mechanisms of secondary injury that occur after spinal cord injury have been elucidated, $[11,21,33]$ it has become apparent that loss of spinal cord microcirculation, loss of autoregulation, and ischemia play important roles in the pathogenesis of secondary spinal cord damage. $[16,17,34]$ It is our hypothesis that aggressive volume and blood pressure augmentation improves spinal cord blood flow, reduces the likelihood of secondary injury, and, when combined with surgical decompression of the spinal cord if indicated, can improve outcome after acute spinal cord injury. The results of this prospective pilot study are encouraging and worthy of further investigation in a controlled, randomized trial.

\section{CLINICAL MATERIAL AND METHODS}

Since 1992 our group has prospectively treated 45 patients with acute cervical spinal cord injuries and 32 patients with acute thoracic spinal cord injuries with early, aggressive medical resuscitation, volume expansion, and elevation and maintenance of MABP to more than $85 \mathrm{~mm} \mathrm{Hg}$ in an attempt to maximize spinal cord perfusion after acute injury.

All patients were initially managed with immobilization, rapid diagnosis, examination by two physicians, rapid triage to the neurosurgical intensive care unit (NICU), and fluoroscope-guided fracture-dislocation reduction as indicated individually according to our established protocol (Fig. 1). 


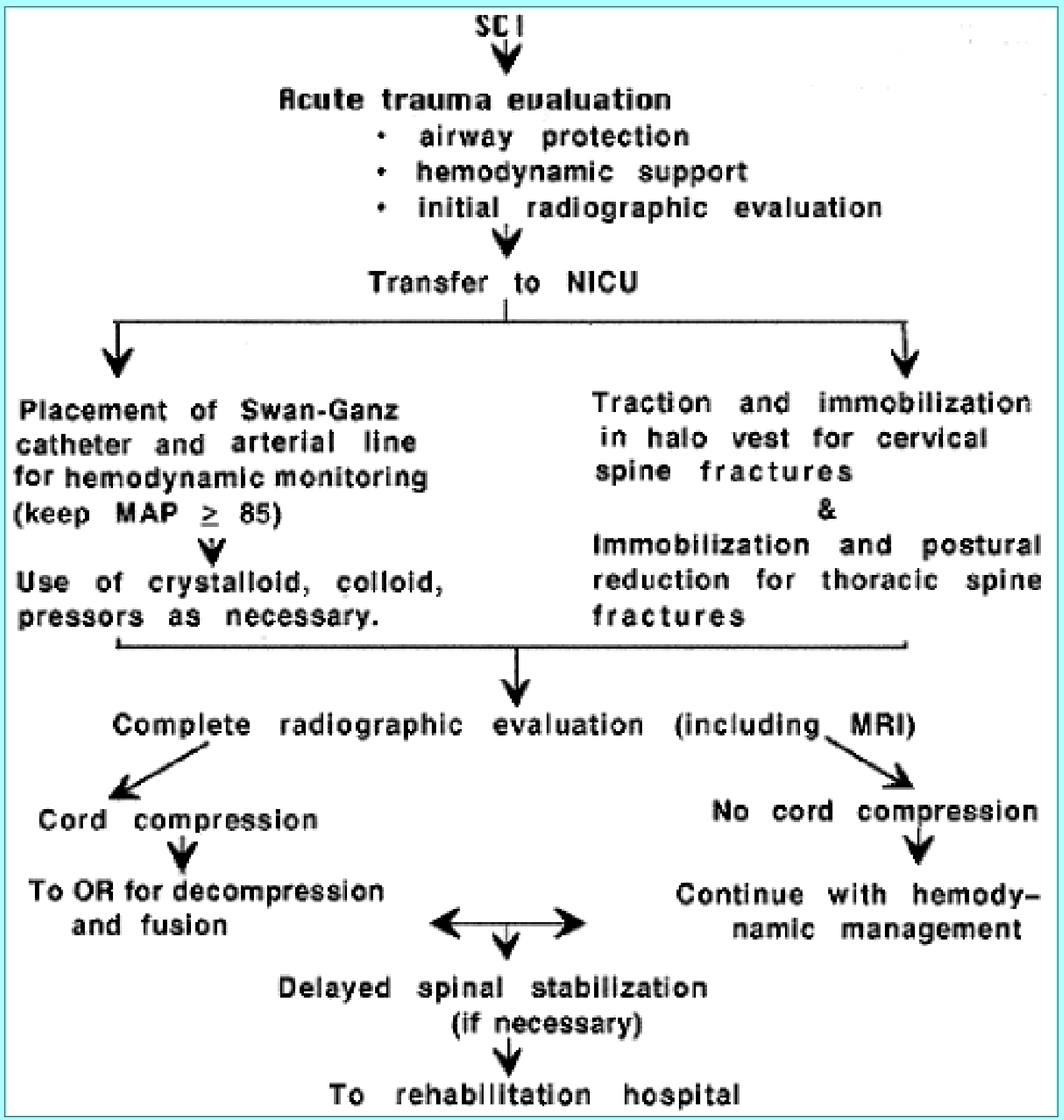

Fig. 1. Management protocol for treatment of acute spinal cord injury (SCI). MAP = mean arterial blood pressure; $\mathrm{MRI}=$ magnetic resonance imaging; $\mathrm{OR}=$ operating room.

All patients were treated with volume resuscitation and administration of vasopressors, as needed, based on each patient's hemodynamic profile, which was determined with the aid of Swan-Ganz and arterial blood pressure catheters to maintain MABP above $85 \mathrm{~mm} \mathrm{Hg}$. All patients were treated with methylprednisolone according to the National Acute Spinal Cord Injury Study II protocol.[2,3] Early fracture-dislocation reduction and cord decompression were accomplished by closed or open means, as necessary, depending on patient examination, medical stability, and radiographic and magnetic resonance (MR) imaging data. Surgery was delayed only in cases of patients with spinal column instability. We did not include a "control" group in this preliminary investigation because our goal was to identify the potential for significant improvement in neurological recovery after traumatic spinal cord injury when using aggressive medical treatment and to compare our results with those expected on the basis of 
"historical" experience in patients with acute spinal cord injury who were managed without aggressive volume and blood pressure augmentation. Patients were managed in this fashion in the NICU regardless of their need for surgery.

On admission, neurological assessment of each patient was independently performed by two physicians and serially thereafter per the protocol until 18 months postinjury. Neurological assessments of patients were made at various time points using the American Spinal Injury Association (ASIA) Impairment Scale[41] (Table 1), determination of ambulatory capacity, and evaluation of bladder function. This combination of neurological assessments was used to provide a more uniform and comprehensive documentation of neurological dysfunction and potential neurological recovery than that provided using standard Frankel or modified Frankel scoring schemes.[5]

\begin{tabular}{|c|c|c|}
\hline \multicolumn{3}{|c|}{$\begin{array}{c}\text { TABLE } 1 \\
\text { AMERICAN SPINAL INJURY ASSOC KTION IMPARMENT SCALE } \\
\text { OF SPINAL CORD INWRIES }\end{array}$} \\
\hline $\begin{array}{l}\text { AGA } \\
\text { Grade }\end{array}$ & $\begin{array}{l}\text { Type of } \\
\text { Iri ury }\end{array}$ & Defirition of Type of Iriury \\
\hline $\begin{array}{l}\text { A } \\
\mathrm{B}\end{array}$ & $\begin{array}{l}\text { complete } \\
\text { incomplete }\end{array}$ & $\begin{array}{l}\text { no motor or sensory function } \\
\text { sensory but no motor function preser ved belo w } \\
\text { le vel of injury }\end{array}$ \\
\hline $\mathrm{C}$ & incomplete & $\begin{array}{l}\text { motor function preser 'ed, but m ajority of musdes } \\
\text { have musde grade }<3\end{array}$ \\
\hline $\mathrm{D}$ & incomplete & $\begin{array}{l}\text { motor function preser ved \& m ajority of musdes have } \\
\text { musde grade } \equiv 3\end{array}$ \\
\hline $\mathrm{E}$ & none & motor \& sensory function normal \\
\hline
\end{tabular}

Patients were excluded from participation in this study if they had a spinal column injury located below $\mathrm{T}-12$ or an associated head injury that precluded neurological assessment of spinal cord function (two patients), if the initial neurological examinations revealed deficits in fewer than five muscle groups with individual muscle group scores greater than or equal to three points (maximum allowable motor index score [MIS] of 85), or if the patient arrived to our institution more than 36 hours after injury occurred.

Follow-up examinations at 6-, 12-, and 18-month intervals were performed in each patient independently by two physicians (A.B.J. and M.N.H.). The mean duration of follow up for patients in this review was 17 months (range 12-24 months).

\section{RESULTS}

To date, 64 patients with acute spinal cord injuries have been followed for 12 months or longer after injury and treatment. Motor vehicle accidents were the most common cause of injury. There were 35 patients with cervical spinal cord injuries (10 with complete neurological injuries and 25 with incomplete injuries) and 29 patients with thoracic spinal cord injuries ( 21 with complete neurological injuries and eight with incomplete injuries at T-12 or above).

\section{Cervical Spinal Cord Injuries}

The 35 patients with cervical spinal cord injuries have been followed at least 12 months after injury and treatment. There were 29 males and six females in this group. Nine patients sustained severe multisystem trauma including one case of minor head injury, five cases of chest injuries, two cases of abdominal injuries requiring laparotomy, and three cases of long bone-pelvic fractures. Of the 10 patients with 
complete neurological injuries the most common vertebral levels of injury were C4-5, C5-6, and C6-7. The most common level of injury in the 25 patients with incomplete cervical injury was C5-6 (Table 2).

\begin{tabular}{|c|c|c|}
\hline $\begin{array}{l}\text { CAUSE AND LEVEL } \\
\text { NON PENE }\end{array}$ & $\begin{array}{l}\text { ABLE } 2 \\
\text { ETAL DAMGGE IN } \\
\text { SPINAL CORD }\end{array}$ & $\begin{array}{l}\text { TIENTS WITH } \\
\text { ES }\end{array}$ \\
\hline & No. of Patients & pinal Cord Iriury \\
\hline Type of Irituy & Complete (\%) & Incomplete (\%) \\
\hline $\begin{array}{l}\text { cervical card injury } \\
\text { cause of injury }\end{array}$ & & \\
\hline $\begin{array}{l}\text { motor wehide accident } \\
\text { fall }\end{array}$ & $\begin{array}{l}6(60) \\
3(30)\end{array}$ & $\begin{aligned} 17(68) \\
5(20)\end{aligned}$ \\
\hline sport injury & $1(10)$ & \\
\hline $\begin{array}{l}\text { motorcycle accident } \\
\text { di ung }\end{array}$ & $\begin{array}{l}0(0) \\
0(0)\end{array}$ & $\begin{array}{l}1(4) \\
2(8)\end{array}$ \\
\hline level of injury & & \\
\hline $\begin{array}{l}C 3-4 \\
C 4-5\end{array}$ & $\begin{array}{l}1(10) \\
3(30)\end{array}$ & $\begin{array}{l}5(20) \\
5(20)\end{array}$ \\
\hline$\frac{\infty}{\infty}-6$ & $\begin{array}{l}330) \\
3300\end{array}$ & $7(28)$ \\
\hline $\mathrm{C}-\mathrm{T} 1$ & 0 (0) & $2(8)$ \\
\hline $\begin{array}{l}\text { tharacic card anjory } \\
\text { cause of injury }\end{array}$ & & \\
\hline $\begin{array}{l}\text { motor wehide accident } \\
\text { fall }\end{array}$ & $\begin{aligned} 16(76) \\
4(19)\end{aligned}$ & $\begin{array}{l}7(88) \\
1(12)\end{array}$ \\
\hline $\begin{array}{l}\text { other } \\
\text { level of injury }\end{array}$ & $1(5)$ & $0(0)$ \\
\hline $\begin{array}{l}\text { level of injury } \\
T-4\end{array}$ & & \\
\hline$T-5$ & $1(5)$ & $\begin{array}{l}0(0) \\
0(0)\end{array}$ \\
\hline$T-6$ & $1(5)$ & $0(0)$ \\
\hline $\mathrm{T}-7$ & $4(19)$ & $0(0)$ \\
\hline $\begin{array}{l}T-8 \\
T-9\end{array}$ & $\begin{array}{l}4(19) \\
1(5)\end{array}$ & $\begin{array}{l}0(0) \\
1(12)\end{array}$ \\
\hline $\mathrm{T}-11$ & $2(10)$ & 2 (25) \\
\hline $\mathrm{T}-12$ & $5(24)$ & $5(63)$ \\
\hline
\end{tabular}

The mean MIS for patients with complete cervical cord injuries (ASIA Grade A) on initial examination was 15 (range 2-40) (Table 3). No patient in this group had bladder function, rectal sphincter tone, or ambulatory capacity on admission. The average MABP for patients with complete cervical cord injuries on arrival at the NICU was $66 \mathrm{~mm} \mathrm{Hg}$ (range $48-82 \mathrm{~mm} \mathrm{Hg}$ ). Four of 10 patients presented with signs of neurogenic shock. Nine patients with complete injuries required administration of vasopressors in addition to volume resuscitation to maintain MABP above $85 \mathrm{~mm} \mathrm{Hg}$. Patients with complete cervical cord injuries were treated in the NICU for an average of 13 days (range 6-39 days). 


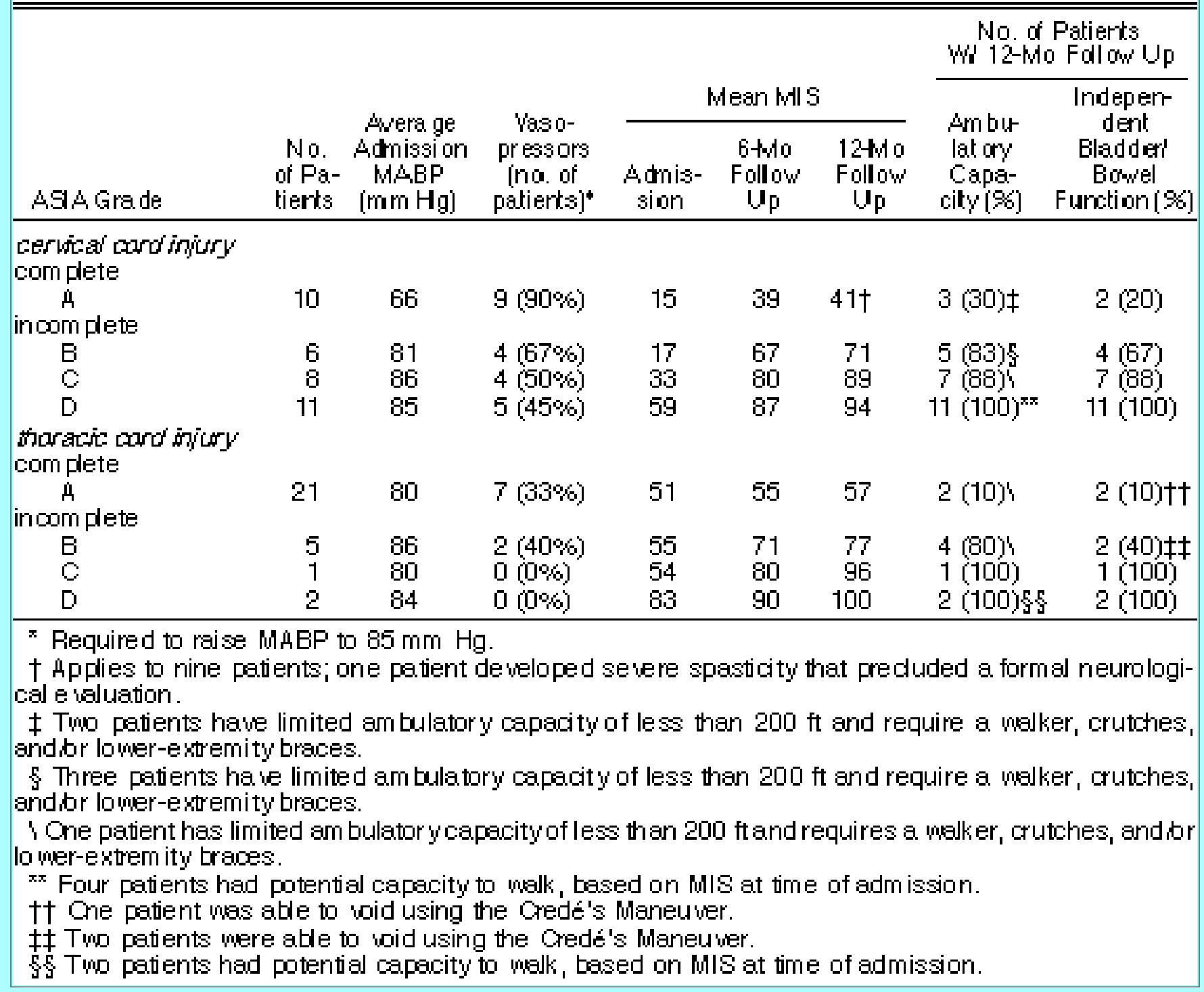

Initial neurological assessment revealed a mean MIS of 44 for the 25 patients with incomplete cervical spinal cord injuries. The mean MIS for ASIA Grade B patients was 17 (range 7-35); for ASIA Grade C patients, 33 (range 7-62); and for ASIA Grade D patients, 59 (range 35-80) (Table 3). No patient had bladder function on admission, although 10 had some measure of rectal sphincter tone, as determined by digital examination. Four patients with incomplete cervical spinal cord injuries had an initial MIS compatible with ambulatory function on admission; all were ASIA Grade D patients with severe central spinal cord injuries. The average MABP for patients with incomplete cervical spinal cord injuries on arrival at the NICU was $83 \mathrm{~mm} \mathrm{Hg}$ (range $60-103 \mathrm{~mm} \mathrm{Hg}$ ). No patient in this group exhibited signs of neurogenic shock. Fifty-two percent of patients (13 patients) with incomplete neurological injuries required administration of vasopressors to maintain MABP above $85 \mathrm{~mm} \mathrm{Hg}$. The initial MABP for the six ASIA Grade B patients was $81 \mathrm{~mm} \mathrm{Hg}$; four required vasopressors. Of eight ASIA Grade C patients with an initial MABP of $86 \mathrm{~mm} \mathrm{Hg}$, four required pressor agents. The average MABP for 11 ASIA Grade D patients was $85 \mathrm{~mm} \mathrm{Hg}$; five of these patients required vasopressors. The average length of stay in the NICU for patients with incomplete cervical spinal cord injuries was 7 days (range 4-21 days).

\section{Thoracic Spinal Cord Injuries}


A follow-up period of 12 months or longer has been accomplished in 29 patients with thoracic spinal cord injuries. There were 25 males and four females in this group. Multiple organ system injuries occurred in 16 patients; the majority of these were severe chest (14 patients) and abdominal injuries (six patients) requiring intensive medical management, surgery, or both. Twenty-one patients had complete neurological injuries on admission (ASIA Grade A). Eight patients had incomplete thoracic spinal cord injuries: five were ASIA Grade B, one ASIA Grade C, and two ASIA Grade D. The most common level of vertebral column injury in patients with thoracic spinal cord injury was T-12 (10 patients [34\%]) (Table 2).

Patients with complete (ASIA Grade A) thoracic spinal cord injury had a mean MIS of 51 (range 50-54) on initial examination (Table 3). No patient had bladder function, rectal tone, or the ability to walk. The initial average MABP for this group was $80 \mathrm{~mm} \mathrm{Hg}$ (range 60-100 $\mathrm{mm} \mathrm{Hg}$ ). Seven (33\%) of these patients required vasopressor therapy in addition to volume resuscitation to maintain MABP above 85 $\mathrm{mm} \mathrm{Hg}$. Blood products for resuscitation were more commonly used in this group than in cervical cord-injured patients, attesting to the severity of the associated chest and abdominal injuries that patients with thoracic spinal cord injury sustained. The mean length of stay in the NICU for patients with complete thoracic spinal cord injury was 15 days (range 7-45 days).

Patients with incomplete thoracic spinal cord injuries had a initial mean MIS of 62. Five ASIA Grade B patients had an initial mean MIS of 55 (range 50-62). The single ASIA Grade C patient had an initial MIS of 54 and the mean MIS of two ASIA Grade D patients was 83 (82 and 84) (Table 3). No patient had bladder function on admission, although four had some rectal sphincter tone, as determined by digital examination. Only two patients, both classified ASIA Grade D, had neurological function and an MIS compatible with walking. The initial average MABP in patients with incomplete thoracic spinal cord injury was $85 \mathrm{~mm} \mathrm{Hg}$ (range $80-100 \mathrm{~mm} \mathrm{Hg}$ ). Only two patients with incomplete thoracic spinal cord injuries, both ASIA Grade B patients, required administration of vasopressors to maintain MABP above $85 \mathrm{~mm} \mathrm{Hg}$. The average length of stay in the NICU for patients with incomplete thoracic spinal cord injuries was 7 days (range 5-13 days).

\section{Surgical Treatment}

Surgical treatment was used in 31 of the 35 patients with cervical cord injuries and 27 of the 29 patients with thoracic spinal cord injuries. Early surgery, defined as occurring within 24 hours of injury, was performed in seven of 35 cervical cord-injured patients and four of 29 thoracic cord-injured patients for spinal cord decompression. Nine patients with cervical and four with thoracic cord injuries underwent surgery between 24 and 72 hours of injury for cord decompression, vertebral column realignment, or spinal stabilization. Delayed surgery, defined as surgery initiated more than 72 hours after injury, was performed on 15 cervical and 19 thoracic cord-injured patients for spinal stabilization and fusion (Table 4). The mean duration from the time of injury to surgery in this latter group was 11 days for the cervical cord injury group (range 4-34 days) and 10 days for the thoracic cord injury group (range 4-34 days). 


\begin{tabular}{|c|c|c|}
\hline \multirow[b]{2}{*}{ Timing \& Method of Strgery } & \multicolumn{2}{|c|}{$\begin{array}{l}\text { No. of Patients Wi } \\
\text { Spinal Cord Iniury }\end{array}$} \\
\hline & Complete [\%] & Incomplete (\%) \\
\hline \multicolumn{3}{|l|}{ cervical cardinjury } \\
\hline surgical inter vention & $8(80)$ & $23(92)$ \\
\hline 24 hrs & & \\
\hline $24-72$ hrs & 2 & 7 \\
\hline$>72 \mathrm{hrs}$ & 3 & 12 \\
\hline \multicolumn{3}{|l|}{ statilization method } \\
\hline anterior cervical fusion & $7(88)$ & $17(74)$ \\
\hline posterior cervical fusion & $1(12)$ & $2(9)$ \\
\hline combined anterior \& posterior fusion & $0(0)$ & $4(17)$ \\
\hline \multicolumn{3}{|l|}{ tharacic card onjory } \\
\hline surgical inter vention & $19(90)$ & $8(100)$ \\
\hline $24 \mathrm{hrs}$ & & \\
\hline $24-72 \mathrm{hrs}$ & 2 & 2 \\
\hline$>72 \mathrm{hrs}$ & 15 & 4 \\
\hline \multicolumn{3}{|l|}{ stabilization method } \\
\hline anterior decom pression fusion & $3(16)$ & $2(25)$ \\
\hline posterior decom pression fusion & $15(79)$ & $6(75)$ \\
\hline com tined anterior \& posterior fusion & $1(5)$ & $0(0)$ \\
\hline
\end{tabular}

\section{Functional Outcome}

There were no recognized untoward effects of aggressive volume resuscitation or MABP elevation therapy. Specifically, there was no instance of hypertensive hemorrhage, stroke, myocardial infarction, or death in this series during a mean follow-up period of 17 months (range 12-24 months).

Neurological recovery after spinal cord injury was variable and typically incomplete. At the 12-month follow-up examination, patients with complete cervical spinal cord injuries had a mean MIS of 41 (range 12-80). Three (30\%) of 10 patients in this group regained ambulatory function (two patients have limited ambulatory capacity of less than $200 \mathrm{ft}$ and require crutches and/or a walker for assistance). Two patients regained voluntary bladder and bowel function (Table 3). Of 10 patients with ASIA Grade A on admission, four remained a Grade A, one patient improved to Grade B, two improved to Grade C, and three were at Grade D at the last follow up (Table 5). No patient in this group who presented with symptoms and signs of neurogenic shock had a meaningful change in function. 


\begin{tabular}{|c|c|c|c|c|c|c|}
\hline \multicolumn{7}{|c|}{$\begin{array}{c}\text { TABLE } 5 \\
\text { OUTCOME IMPROVEMENT IN 64 PATIENTS WITH SPINAL CORD INURV } \\
\text { ACCORD NG TO ASIA IMPAIRMENT SCALE }\end{array}$} \\
\hline \multirow[b]{2}{*}{ Iribal ASAGrade } & \multirow{2}{*}{$\begin{array}{l}\text { No. of } \\
\text { Patierts }\end{array}$} & \multicolumn{5}{|c|}{ Outcome ASA Grade at 12-wo Follow Up } \\
\hline & & A & $\mathrm{B}$ & $\mathrm{C}$ & D & $\mathrm{E}$ \\
\hline \multicolumn{7}{|l|}{ cervical cord injury } \\
\hline A & 10 & 4 & 1 & 2 & 3 & - \\
\hline $\mathrm{B}$ & 6 & - & 1 & 1 & 4 & - \\
\hline $\mathrm{C}$ & 8 & - & - & 1 & 4 & 3 \\
\hline D & 11 & - & - & - & 6 & 5 \\
\hline \multicolumn{7}{|l|}{ thoracic cord injury } \\
\hline A & 21 & 14 & 2 & 3 & 2 & - \\
\hline $\mathrm{B}$ & 5 & - & 1 & 2 & 2 & - \\
\hline $\mathrm{C}$ & 1 & - & - & - & 1 & - \\
\hline D & 2 & - & - & - & - & 2 \\
\hline
\end{tabular}

Patients with incomplete cervical cord injuries had a mean MIS of 85 (range 52-100) 12 months postinjury. Grade B patients had a mean MIS of 71 (range 52-92), Grade C patients had a mean MIS of 89 (range 62-100), and Grade D patients improved to a mean MIS of 94 (range 74-100). Twenty-three (92\%) of 25 patients with incomplete cervical cord injuries had the ability to walk 1 year after injury (four patients have limited ambulatory capacity of less than 200 feet and require crutches and/or walker for assistance); only four of these patients had an initial MIS consistent with ambulation. Twenty-two (88\%) of 25 patients regained bladder function. A comparison of outcome ASIA grades and initial ASIA injury grades is depicted in Table 5.

Patients with complete thoracic spinal cord injuries had a mean MIS of 57 at 12-month follow-up evaluation (range 50-94). Only two (10\%) of the 21 patients in this group regained ambulatory function (one patient has limited ambulatory capacity of less than $200 \mathrm{ft}$ ) and functional bladder control. Fourteen ASIA Grade A patients remained a Grade A at their last follow up. Two patients improved to ASIA Grade B, three to ASIA Grade C, and two to ASIA Grade D (Table 5).

Patients with incomplete thoracic spinal cord injuries had a mean MIS of 85 (range 71-100) at 12 months postinjury. The ASIA Grade B patients improved to a mean MIS of 77 (range 71-88). The single ASIA Grade C patient had a MIS of 961 year after injury. Both ASIA Grade D patients had a 12-month follow-up MIS of 100. Five (63\%) of eight patients with incomplete thoracic cord injuries regained useful bladder function. Seven (88\%) of eight patients were walking at 12 months (one patient has limited ambulatory capacity); two of these patients, however, had neurological function and an MIS consistent with walking on admission (ASIA Grade D patients). A comparison of ASIA outcome grades and initial ASIA grades is outlined in Table 5.

\section{Data Analysis}

We found no statistically significant difference between the preoperative neurological examination and the selection for, or timing of, surgery in patients with cervical or thoracic spinal cord injuries in this series (cervical, $\mathrm{p}<0.125$; thoracic, $\mathrm{p}<0.587$ ). Similarly, stratification of neurological recovery at the 12-month follow up for cervical and thoracic spinal cord injuries revealed no statistically significant impact of the timing of surgery with respect to outcome (cervical, $p<0.985$; thoracic, $p<0.352$ [one-way analysis of variance]). 


\section{DISCUSSION}

Advances in transport and triage; radiographic diagnosis; and inpatient hospital care, including immobilization, pharmacology (methylprednisolone), and early fracture-dislocation reduction, appear to improve outcome of acute spinal cord injuries after injury.[1,13,15,32,40] The present medical case management and degree of resuscitation in spinal cord-injured patients in North America is variable and is dependent on the strategies and experiences of individual surgeons and institutions. Although a variety of investigators are attempting to standardize the surgical treatment of these patients, to date no study has validated specific medical treatment regimens.

Volume resuscitation and induced blood pressure elevation, used to maximize cerebral perfusion, is an accepted treatment strategy to optimize outcome among patients suffering cerebral ischemia as a result of subarachnoid hemorrhage, stroke, and closed head injury.[24,25,28-31] Many of the same secondary injury mechanisms that occur in the brain after ischemia or trauma are thought to occur in the spinal cord after acute injury.[19] Volume expansion and blood pressure augmentation, including relative hemodilution, may serve to maximize spinal cord blood flow and perfusion after traumatic injury. It is our hypothesis that the ideal management of the patient with acute spinal cord injury is not pharmacological therapy, early fracture reduction, or surgery, but a combination of the three plus early, aggressive volume resuscitation and blood pressure elevation to maximize spinal cord perfusion.

Swan-Ganz and indwelling arterial catheters were used in each patient to monitor individual hemodynamics, assess responses to therapy, and guide treatment as necessary to maximize each patient's cardiopulmonary performance. No important complications were recognized.

We do not know the ideal MABP necessary to maximize spinal cord perfusion after acute injury. We are not aware of a way to measure spinal cord perfusion in the clinical setting. We arbitrarily selected an MABP above $85 \mathrm{~mm} \mathrm{Hg}$ as the target MABP in this pilot study. Treatment was instituted as early as possible after injury in each patient to maintain MABP above $85 \mathrm{~mm} \mathrm{Hg}$ throughout the course of treatment. Although an MABP of $85 \mathrm{~mm} \mathrm{Hg}$ is not considered to be in the hypertensive range, it is considered to be "high normal" compared with established normative blood pressure values for noninjured males aged 15 to 30 years.[20] Although the selection of $85 \mathrm{~mm} \mathrm{Hg}$ for the target MABP was empirical, it was based on our previous experience with patients suffering cerebral ischemia. An MABP of $85 \mathrm{~mm} \mathrm{Hg}$ was found to be effective in the successful management of patients with moderate head injury we have treated who had intracranial pressure elevations to the level of $15 \mathrm{~mm} \mathrm{Hg}$.[29]

Acute resuscitation was accomplished initially using crystalloid. Blood products were administered to maintain a hematocrit above $32 \%$, ideally one between $33 \%$ and $37 \%$. Once the Swan-Ganz catheter was in place, further resuscitation was dictated by individual patient hemodynamic parameters. Vasopressor therapy was added when volume resuscitation was adequate but MABP remained at or below $85 \mathrm{~mm} \mathrm{Hg}$ (Fig. 1). Dopamine (2.5-5 $\mu \mathrm{g} / \mathrm{kg} / \mathrm{minute}$ ), followed by Levophed if needed (0.01-0.2 $\mu \mathrm{g} / \mathrm{kg} / \mathrm{minute}$ ), was administered intravenously and titrated to maintain an MABP greater than $85 \mathrm{~mm} \mathrm{Hg}$.

Therapy was continued irrespective of the need for, or timing of, surgery, including surgery for chest, abdominal, or long-bone injuries for 7 days in the NICU setting. A duration of 7 days was selected as the minimum duration of treatment in symptomatic patients, based on data from experimental spinal cord injury studies, which indicate that maximum cord edema and spinal cord vascular congestion occur between 3 and 5 days after spinal cord injury.[42] The patients were weaned from therapy earlier than 7 
days postinjury if they maintained an MABP over $85 \mathrm{~mm} \mathrm{Hg}$ without administration of vasopressors and if they were medically stable with respect to any associated injury.

The decision to perform surgery on a given patient was based on individual patient characteristics including medical status and stability after multiple organ system trauma; the MR appearance of intrinsic spinal cord injury versus compression by fracture dislocation, bone, disc, or hematoma; failure of closed reduction or rigid external immobilization; and the presence of spinal instability unlikely to heal over time in a rigid orthosis. Eleven patients were surgically treated within 24 hours of injury and 13 patients underwent surgery between 24 and 72 hours postinjury for spinal cord decompression and/or reduction of a fracture-dislocation injury, followed by stabilization and fusion. The only distinction between patients undergoing surgery within 24 hours of injury and those treated between 24 and 72 hours postinjury was the patient's medical status after sustaining trauma and resuscitation and/or the failure of external immobilization to maintain vertebral column realignment without cord compression. There was no difference in the severity of neurological injury between the two groups.

Patients in the delayed surgery group had spinal instability without cord compression, the MR appearance of either cord transection or severe intrinsic cord injury for which surgical decompression was not indicated, or severe life-threatening associated organ system injuries that put the patient at an unacceptable medical risk until stabilized.

The majority of patients in this study demonstrated improvement in neurological function at 6 and 12 months follow up. Four patients with complete cervical cord injuries, all of whom presented in neurogenic shock suggestive of autonomic disruption, and 14 patients with complete thoracic cord injuries manifested no meaningful recovery at 12 months postinjury despite aggressive combined therapy. The MR appearance of the spinal cord in these patients was consistent with either transection of the spinal cord or severe intrinsic spinal cord injury. The patients with thoracic cord injuries, in particular, had severe unstable chest and abdominal injuries in addition to their thoracic vertebral column translocation injuries with or without thoracic cord transection, indicative of the significant traumatic forces these patients experienced. Of note is that no patient with a complete thoracic spinal cord injury above the T-9 level regained functional motor recovery at their 1-year follow-up evaluation.

Six patients presenting with complete cervical cord injuries were improved as of the 12-month follow up. Five of six patients had a meaningful return of function: two regained useful bladder and bowel function and three the ability to walk (two of three require crutches or a walker).

Improvement in neurological function among patients with incomplete cervical cord injuries in this study was more consistent than that identified for patients with complete cervical cord injuries. One ASIA Grade B patient remained Grade B and one ASIA Grade C patient remained Grade C. All 23 others were improved as of their 12-month follow up. Twenty-two patients $(88 \%)$ regained useful bladder and bowel function and 23 (92\%) could walk (four require crutches or a walker) as of the last follow up. Only four of these patients had neurological function and an MIS consistent with walking at the time of admission.

At 12 months postinjury, neurological outcome in patients who sustained thoracic spinal cord injuries was not as good as that observed in patients who suffered cervical spinal cord injuries. Thoracic spinal cord injuries are not isolated injuries. In general, these patients sustain more severe trauma than patients with cervical cord injuries, have greater cardiopulmonary dysfunction, and poorer, more tenuous blood supply to the site of the injured spinal cord (watershed). Only seven patients with complete thoracic cord injuries showed improvement at the last follow up. Five had a meaningful return of function, two of 
whom regained useful bladder function and the ability to walk. Patients with incomplete thoracic cord injuries, typically those with injuries between T-9 and T-12, generally improved. Five (73\%) of eight patients in this group regained useful bladder function and seven $(88 \%)$ of eight regained the ability to walk (one requires assistance).

A search of the literature shows that the neurological recovery of patients with complete spinal cord injuries managed with aggressive volume resuscitation and augmentation of blood pressure in this series is consistently better than that reported for other published series of patients with complete cervical and/or thoracic spinal cord injuries.[7,8,10,23,35,38] The improvements identified at the 12-month follow up in patients in our study with incomplete cervical and thoracic cord injuries is even more dramatic. $[4,7,22,23,36,37,39]$ The enhanced neurological outcome observed after spinal cord injury in this study was in addition to, and/or distinct from, any potential benefit provided by surgery.

We conclude that addition of volume resuscitation and blood pressure augmentation to the management regimen of patients with spinal cord injuries improves spinal cord perfusion and improves neurological outcome after acute traumatic injury. It is a treatment modality that can be initiated immediately after injury (even by nonneurosurgical health care providers) and can be continued regardless of the need for patient transfer to a definitive care facility or the need for surgery. A multicenter, randomized trial is warranted to characterize the ideal target MABP in the patient suffering from acute spinal cord injury and to determine the optimal duration of combined aggressive medical-surgical treatment.

\section{References}

1. Benzel EC, Larson SJ: Functional recovery after decompressive spine operation for cervical spine fractures. Neurosurgery 20:742-746, 1987

2. Bracken MB, Holford TR: Effects of timing of methylprednisolone or naloxone administration on recovery of segmental and long-tract neurological function in NASCIS 2. J Neurosurg 79:500-507, 1993

3. Bracken MB, Shepard MJ, Collins WF, et al: A randomized, controlled trial of methylprednisolone or naloxone used in the treatment of acute spinal-cord injury. Results of the Second National Acute Spinal Cord Injury Study. N Engl J Med 322:1405-1411, 1990

4. Bradford DS, Mcbride GG: Surgical management of thoracolumbar spine fractures with incomplete neurologic deficits. Clin Orthop 218:201-216, 1987

5. Capaul M, Zollinger H, Satz N, et al: Analyses of 94 consecutive spinal cord injury patients using ASIA definition and modified Frankel score classification. Paraplegia 32:583-587, 1994

6. Dolan EJ, Tator CH, Endrenyl L: The value of decompression for acute experimental spinal cord compression injury. J Neurosurg 53:749-755, 1980

7. Donovan WH, Cifu DX, Schotte DE: Neurological and skeletal outcomes in 113 patients with closed injuries to the cervical spinal cord. Paraplegia 30:533-542, 1992

8. Ducker TB, Lucas JT, Wallace CA: Recovery from spinal cord injury. Clin Neurosurg 30:495-513, 1983 
9. Duh MS, Shepard MJ, Wilberger JE, et al: The effectiveness of surgery on the treatment of acute spinal cord injury and its relation to the pharmacological treatment. Neurosurgery 35:240-248, 1994

10. Frankel HL, Hancock DO, Hyslop G, et al: The value of postural reduction in the initial management of closed injuries of the spine with paraplegia and tetraplegia. Paraplegia 7:179-192, 1969

11. Fried LC, Goodkin R: Microangiographic observations of the experimentally traumatized spinal cord. J Neurosurg 35:709-714, 1971

12. Geisler FH, Dorsey FC, Coleman WP: Recovery of motor function after spinal-cord injury--a randomized, placebo-controlled trial with GM-1 ganglioside. N Engl J Med 324:1829-1838, 1991

13. Gillingham J: Early management of spinal cord trauma. J Neurosurg 44:766-767, 1976 (Letter)

14. Guha A, Tator CH, Endreni L, et al: Decompression of the spinal cord improves recovery after acute experimental spinal cord compression injury. Paraplegia 25:324-339, 1987

15. Hadley MN, Fitzpatrick BC, Sonntag VKH, et al: Facet fracture-dislocation injuries of the cervical spine. Neurosurgery 30:661-666, 1992

16. Hall ED, Wolf DL: A pharmacological analysis of the pathophysiological mechanisms of posttraumatic spinal cord ischemia. J Neurosurg 64:951-961, 1986

17. Harris $\mathrm{P}$, Karmi MZ, McClemont E, et al: The prognosis of patients sustaining severe cervical spine injury $\left(\mathrm{C}_{2}-\mathrm{C}_{7}\right.$ inclusive $)$. Paraplegia 18:324-330, 1980

18. Heiden J, Weiss MH, Rosenberg AW, et al: Management of cervical spine cord trauma in Southern California. J Neurosurg 43:732-736, 1975

19. Isaac L, Pejic L: Secondary mechanisms of spinal cord injury. Surg Neurol 43:484-485, 1995

20. Joint National Committee: The Fifth Report of the Joint National Committe on detection, evaluation and treatment of high blood pressure (JNCV). Arch Intern Med 153:154-183, 1993

21. Kobrine AI, Doyle TF, Rizzoli HV: Spinal cord blood flow as affected by changes in the systemic arterial blood pressure. J Neurosurg 44:12-15, 1976

22. Krengel WF III, Anderson PA, Henley MB: Early stabilization and decompression for incomplete paraplegia due to a thoracic-level spinal cord injury. Spine 18:2080-2087, 1993

23. Levi L, Wolf A, Rigamonti D, et al: Anterior decompression in the cervical spine trauma: does the timing of surgery affect the outcome? Neurosurgery 29:216-222, 1991

24. Levy ML, Day JD, Zelman V, et al: Cardiac performance enhancement and hypervolemic therapy. Neurosurg Clin North Am 5:725-739, 1994

25. Levy ML, Giannotta SL: Cardiac performance indices during hypervolemic therapy for cerebral vasospasm. J Neurosurg 75:27-31, 1991

26. Rivlin AS, Tator CH: Effect of duration of acute spinal cord compression in a new acute cord injury model in the rat. Surg Neurol 10:39-43, 1978 
27. Rosner MJ, Coley IB: Cerebral perfusion pressure, intracranial pressure, and head elevation. J Neurosurg 65:636-641, 1986

28. Rosner MJ, Daughton S: Cerebral perfusion pressure management in head injury. J Trauma 30:933-941, 1990

29. Rosner MJ, Rosner SD, Johnson AH: Cerebral perfusion pressure: management protocol and clinical results. J Neurosurg 83:949-962, 1995

30. Solomon RA, Fink ME, Lennihan L: Early aneurysm surgery and prophylactic hypervolemic hypertensive therapy for the treatment of aneurysmal subarachnoid hemorrhage. Neurosurgery 23:699-704, 1988

31. Solomon RA, Fink ME, Lennihan L: Prophylactic volume expansion therapy for the prevention of delayed cerebral ischemia after early aneurysm surgery. Results of a preliminary trial. Arch Neurol 45:325-332, 1988

32. Sussman BJ: Early mangement of spinal cord trauma. J Neurosurg 44:766, 1976 (Letter)

33. Tator $\mathrm{CH}$, Fehlings MG: Review of the secondary injury theory of acute spinal cord trauma with emphasis on vascular mechanisms. J Neurosurg 75:15-26, 1991

34. Wallace MC, Tator $\mathrm{CH}$, Frazee P: Relationship between posttraumatic ischemia and hemorrhage in the injured rat spinal cord as shown by colloidal carbon angiography. Neurosurgery 18:433-439, 1986

35. Waters RL, Adkins RH, Yakura JS, et al: Motor and sensory recovery following complete tetraplegia. Arch Phys Med Rehabil 74:242-247, 1993

36. Waters RL, Adkins RH, Yakura JS, et al: Motor and sensory recovery following incomplete paraplegia. Arch Phys Med Rehabil 75:67-72, 1994

37. Waters RL, Adkins RH, Yakura JS, et al: Motor and sensory recovery following incomplete tetraplegia. Arch Phys Med Rehabil 75:306-311, 1994

38. Waters RL, Yakura JS, Adkins RH, et al: Recovery following complete paraplegia. Arch Phys Med Rehabil 73:784-789, 1992

39. Wiberg J, Hauge HN: Neurological outcome after surgery for thoracic and lumbar spine injuries. Acta Neurochir 91:106-112, 1988

40. Wolf A, Levi L, Mirvis S, et al: Operative management of bilateral facet dislocation. J Neurosurg 75:883-890, 1991

41. Yarkony GM, Chen D: Rehabilitation of patients with spinal cord injury, in Braddom RL (ed): Physical Medicine \& Rehabilitation. Philadelphia: WB Saunders, 1996, pp 1149-1172

42. Yashon D, Bingham GW Jr, Faddoul EM, et al: Edema of the spinal cord following experimental impact trauma. J Neurosurg 38:693-697, 1973

Manuscript received November 5, 1998. 
Accepted in final form December 18, 1998.

This article was previously published in the August 1997 issue of the Journal of Neurosurgery.

Address reprint requests to: Mark N. Hadley, M.D., Division of Neurosurgery, University of Alabama at Birmingham, 1813 Sixth Avenue South/511 MEB, Birmingham, Alabama 35294. 